\title{
Travelling on parallel tracks: Patient and physician perspectives on why women delay seeking care for breast cancer symptoms
}

by Leeat Granek, Barbara Fitzgerald, Karen Fergus, Mark Clemons and Ruth Heisey

\section{Abstract}

In the breast cancer literature, "delayed presentation" is defined as a delay of $>3$ months from the self-detection of a new breast cancer symptom until presentation to a health care provider. It has been established that early detection of breast cancer is associated with better health outcomes. The purpose of this qualitative study was to explore reasons why women delay seeking care for their breast cancer symptoms by asking both women and family physicians for their perspectives. Narrative analysis was used to reveal the ways in which people make sense of their experiences and which parts of their stories are most important to them. We found differences in the explanatory narratives of women versus those of family physicians when it came to understanding delay. Suggestions to promote

\section{About the authors

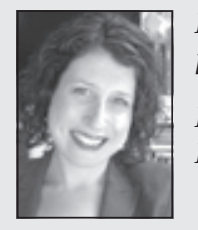 \\ Leeat Granek, Department of Psychology, Hospital for Sick Children (Sick Kids), Toronto, Ontario \\ Leeat Granek, PhD, Hospital for Sick Children (Sick Kids), Toronto, Ontario. Leeatg@gmail.com}

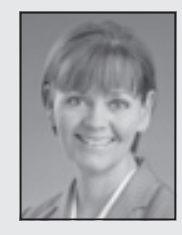

Barbara Fitzgerald, RN, MScN, Director of Nursing, Princess Margaret Hospital, Toronto, Ontario

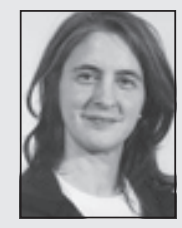

Karen Fergus, PhD, CPsych, Department of Psychology, Sunnybrook Odette Cancer Centre, York University, Toronto, Ontario

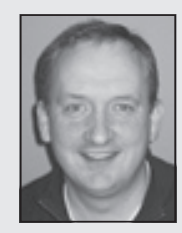

Mark Clemons, FRCP(UK), Division of Medical Oncology, The Ottawa Hospital Cancer Centre, University of Ottawa, Ottawa, Ontario

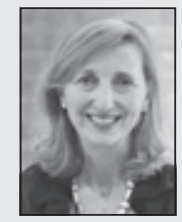

Ruth Heisey, MD, CCFP, FCFP, Family Physician/ GP Oncologist, Women's College Hospital/Princess Margaret Hospital, Clinician Investigator/Associate Professor, University of Toronto, Toronto, Ontario earlier presentation include improving physician-patient communication and encouraging physician and health care professional neutrality in making attributions about women's delay.

\section{Background}

Research in the area of delay in seeking medical care for breast cancer symptoms (e.g., lump, nipple discharge, thickening, inverted nipple) indicates that delays in the detection and treatment of breast cancer are associated with larger tumours and worse survival outcomes (O’Mahoney \& Hegarty, 2009; Richards, Smith, Ramirez, Fentiman, \& Rubens, 1999). In this setting, delay is defined as waiting three months or longer to see a medical professional when first finding what is perceived to be an abnormal breast symptom (Facione, Miaskowski, Dodd, \& Paul, 2002; Richards et al., 1999). Reducing the time between symptom detection and seeking medical consultation could be an important goal in order to produce better clinical outcomes.

Patient characteristics and delay for breast symptoms. Incidences of delay can range from $10 \%$ to $42.5 \%$ (Facione et al., 2002; O'Mahoney \& Hegarty, 2009). Causes of delay are varied and studies have indicated that patient, health care provider, and system related factors have been attributed (Andersen, Cacioppo, \& Roberts, 1995; Arndt et al., 2003; Bish, Ramirez, Burgess, \& Hunter, 2005; Caplan \& Helzlsouer, 1992-1993; Fergus et al., 2011; Granek \& Fergus, 2011). Patient factors described as influencing symptom appraisal and contributing to delay in presentation include: older age, cultural beliefs and attitudes, misperception of the presenting symptom as not being serious, a non-lump presentation, and/or the presence of anxiety (Meechan, Collins, \& Petrie, 2003; O’Mahoney \& Hegarty, 2009). Poor health care utilization habits have been correlated with negative expectations about treatment, concern about cost, pain and uncertainty of cure which impact on decisions to seek care (Caplan \& Helzlsouer, 1992-1993).

Family physicians and delay. Given the association between early presentation and improved health outcomes, the ideal practice from a medical perspective is that a woman presents to her family physician's office quickly upon discovery of an abnormality in her breast. The family physician (FP) can then complete the diagnostic work-up and refer to an oncologist if needed (Beattie, 2009; Davis, 2008).

As the family physician's office is often the first place a woman will access to discuss breast related concerns, FPs are an especially important group to examine for their perspectives on why they think women delay seeking care. There is little data on FPs' perspectives of factors influencing delay in help-seeking in their patients with breast cancer symptoms or strategies used to flag individuals who may be at risk for delay in their practice (De Nooijer, Lechner, \& De Vries, 2001; Heisey, Clemons, Granek et al., 2011). 
Gaps in our understanding. Although several studies have been published looking at women's symptom appraisal and presentation when it comes to breast abnormalities, delay continues to be a significant problem for the health care system. This is especially true for patients with Locally Advanced Breast Cancer (LABC), patients who may have postponed seeking medical consultation for their symptoms and who have particularly poor survival outcomes. The purpose of this study was to explore why women delay seeking care for their breast cancer symptoms by asking both patients and physicians about their perspectives on the matter.

\section{Methods}

\section{Participants}

Patient Informants. A purposive sample of 14 women was recruited. Inclusion criteria entailed women with the following characteristics: English-speaking, a self-detected breast cancer symptom (e.g., lump, nipple discharge, inverted nipple); and waited 3 months or more to present to their family physician. The sample consisted of 14 women diagnosed with breast cancer, currently living in the Greater Toronto Area (GTA) and being treated at a large urban cancer centre in Toronto. The women's disease characteristics ranged from a primary diagnosis of breast cancer to metastatic disease at the time of initial presentation to the oncologist. The average age of the women was 56 years (range $=$ 34 to 67 years). Slightly more than half of the sample was white (60\%). One woman was African-Canadian (7\%), and four women were Asian (28.5\%). At the time of the interview, five (35\%) women had Stage 1 or 2 disease; six women (43\%) had locally advanced breast cancer (LABC) (Stage 3); and three (21\%) women had metastatic disease. The amount of time women delayed seeking help ranged from 3 months to 4.5 years with the average time of delay being 11 months (See Table 1).
Family physician informants. Purposive sampling was used to identify physician informants eligible for inclusion. They must have cared for one or more women in the past five years who presented three months or more after the onset of a breast cancer symptom. The physician sample consisted of 10 FPs (females $=8$, males $=2$ ) recruited from the list of physicians who had referred patients to the study hospital, or by approaching the FPs of our patient informants with their consent. Physicians who agreed to be interviewed were asked to identify suitable colleagues who might be interested and these were also approached. All of the physicians specialized in family medicine and had a range of work experience from $<1-25$ years with the average being 18 years.

\section{Procedures}

Approval was obtained from the appropriate Research Ethics Board. Women were approached by a research nurse, or oncologist on the research team in the LABC and Diagnostic clinics in a large cancer centre in Toronto. If a woman agreed to participate, the research team was notified and followed-up with a call or an in-person meeting in the clinic to schedule an interview. Informed consent and the participant's agreement to the interview being audio-recorded were reconfirmed at the beginning of each interview. After signing a consent form, participant informant interviews were conducted in person $(n=14)$ in the hospital by an experienced qualitative researcher (LG).

Family physician informants were recruited by the physician investigators in this study who sent out a flier about the project via email or by fax to various clinics in the Greater Toronto Area. Physicians who responded to the email or the fax were contacted by the interviewer to set up an interview time. They were interviewed in their offices also by the first author and in

Table 1. Demographics of women

\begin{tabular}{|c|c|c|c|c|}
\hline Ethnicity & Age at diagnosis & Clinic recruited from & Presenting symptom & Delay (months) \\
\hline Caucasian & 51 & $* \mathrm{LABC}$ & Lump & 4 \\
\hline Caucasian & 44 & LABC & Two lumps & 3 \\
\hline Caucasian & 48 & Diagnostic & Nipple discharge and pain & 6 \\
\hline Caucasian & 46 & LABC & Inverted nipple & 12 \\
\hline Asian & 67 & LABC & Lump and pain & 3 \\
\hline Asian & 51 & Diagnostic & Mass and tenderness & 6 \\
\hline Caucasian & 80 & Diagnostic & Inverted nipple & 54 \\
\hline African-Canadian & 40 & LABC & Lump & 24 \\
\hline Caucasian & 42 & Metastatic & Lump & 4 \\
\hline Caucasian & 64 & Metastatic & Two lumps & 9 \\
\hline Asian & 65 & Diagnostic & Lump and pain & 7 \\
\hline Caucasian & 34 & Diagnostic & Indrawing & 3 \\
\hline Caucasian & 75 & Metastatic & Lump & 12 \\
\hline Asian & 74 & LABC & Inverted nipple & 9 \\
\hline
\end{tabular}


two cases by a research assistant. Interviews lasted between 45 minutes to 1.5 hours and were recorded and transcribed. In both groups, a semi-structured interview guide was used. The women were asked to elaborate on their thoughts and feelings as related to seeking help from a medical professional in the case of the breast symptoms (See Appendix A for interview guides). The physicians were asked why they thought women delayed seeking care, whether they felt there was anything they might have done to encourage their patients to seek help sooner, and if they could identify strategies that might have encouraged earlier presentation.

\section{Data analysis}

Data were analyzed in two steps. The first stage of analysis involved using a descriptive thematic analysis (Miles \& Hubermann, 1994). Research team members and co-authors met to read the first five interview transcripts and formulate an initial cross-case coding framework. The data were then examined line-by-line and coded into segments by naming and labelling with words reflecting actions. Nvivo 8 software, a computer-assisted qualitative data management program, was used to code, store, and organize the data. Following the line-by-line analysis, a focused coding process helped to integrate and describe larger segments of data. Codes generated during the early phase of the analysis were more descriptive and reflected the content of the participants' words but, as the analysis progressed, more abstract, higher order themes were generated. The final coding scheme was developed through ongoing discussions with three members of the research team, based on a thorough reading of each transcript. Analysis of the data stopped when the team determined that we had reached saturation and that no more new themes were emerging.

The second stage of the data analysis, and the focus of this paper, involved examination of narratives found in the transcripts. Narrative analysis aims to examine how events are constructed by subjects from their own perspectives, and reveals the ways in which people make sense of their experiences and which parts of their stories are most important to them (Bruner, 1987, 1990; White, 1981). This type of analysis is not a "step by step", linear or prescriptive method, but rather is about focusing on certain aspects of how participants tell their stories.

The larger coding scheme developed previously in the thematic analysis was reorganized to focus on the narrative explanations of physicians and patients when it came to delay. Codes pertaining to reasons women delay seeking care were pulled out of the larger coding scheme and women's explanations were compared to the physician's explanations. In instances where the narratives overlapped-or where the physicians and the patients agreed on the reasons for delay-they were put into the category of Converging Explanations (See Heisey et al., 2011 for a report of these findings). In instances where the narratives diverged-where the physicians' and patients' narratives for delay did not accord with one another, the following categories

Table 2. Patient narratives around explanation for delay

\begin{tabular}{|l|l|}
\hline Doctor-related issues & Person-related issues \\
\hline - Feeling dismissed in past & - Discomfort and squeamishness \\
- Having hard-to-diagnose & about breasts \\
conditions & Reproductive and pregnancy- \\
- Previous breast complaints & related issues \\
dismissed & - Age \\
& - Cultural stigma around breast \\
& cancer \\
\hline
\end{tabular}

were created: Patient Explanations for Delay and Family Physician Explanations for Delay. These divergent explanations are the focus of this paper.

\section{Findings}

Closer examination of the divergent narratives of the women and the physicians inspired the visual metaphor of "Travelling on Parallel Tracks." This metaphor captures the idea that when it comes to delay, family physicians and patients may be missing each other in their explanatory paradigms and travelling along virtual parallel tracks. It is important to stress that this finding does not claim to make assessments about "right" or "wrong", or what the "real truth" is about why women delay seeking care, but rather is a discovery that family physicians and patients may not fully understand each other when it comes to this phenomenon. A description of the women's and the physicians' explanations for delay follows.

\section{Patients' explanations for delay}

The patient informants listed a number of reasons why they avoided seeking care for their breast cancer symptoms that were not mentioned by our physician informants. These entailed two broad themes (See Table 2). The first theme pertained to "doctorrelated issues" such as feeling a general lack of respect during previous doctor-patient encounters, feeling dismissed in the past by a physician for either having a hard-to-diagnose health condition(s), or having a previous breast symptom that was not taken seriously by the doctor. The second theme dealt with "person-related issues" such as a woman being uncomfortable with her breasts; having difficulty attributing significance to her symptoms due to changes in the feel of the breast tissue during pregnancy or in vitro fertilization (IVF) treatment, or due to breastfeeding issues; being "too young" to have breast cancer; and/or to having a cultural stigma around a cancer diagnosis. These findings are further distilled below.

Doctor-related issues. Feeling dismissed in the past by a doctor was named as a reason that women delayed care. If women felt that physicians did not take their medical concerns seriously in the past, they were more likely to avoid the medical care system in the present. One participant who did not feel respected by her FP stated:

103: [with my fibromyalgia and the chronic fatigue], before, years ago when I was first diagnosed... they don't actually say, but they think it's all in your head.... don't tell me... when my legs are hurting and my arms are hurting, it's all in my head.

Related to dismissal was the theme of having hard-to-diagnose health concerns. When patients had particularly complex and ambiguous symptom presentation due to conditions such as fibromyalgia, chronic fatigue syndrome, and irritable bowel syndrome (IBS), the breast symptom was viewed by some participants as moving to the bottom of the priority list for the physician. One participant who presented with a large lump in the LABC clinic, and delayed seeking care for four months noted:

101: The kinds of things in my experience that I suffer from are hard to diagnose. You know if you cut your arm off and you go to the hospital, they can see what needs to be done....I went to a neurologist for my migraines, and I said to the neurologist, I have these migraines, I have them all the time, the pain is excruciating, I see spots.... He said don't worry about it. So basically, I was told that it doesn't count. So I have had several experiences of what I would consider to be useless treatment. So, you know, I don't go. 
Finally, women talked about cases where they felt dismissed by their family physician when they presented specifically with a breast symptom. One woman delayed seeking care for her lump for two years because her doctor had not taken her first (benign) lump seriously. The previous negative experience influenced her comfort level in presenting the next time with symptoms that proved, ultimately, to be malignant. She noted:

109: Anytime I'd go (to the doctor, he) said "you know, breasts are not always the same size," (Yes, you've) already been told, "you have lumpy breasts"... I said, probably it's the same thing going on. So having been dismissed the first time, I said, I'm overreacting, just leave it alone. So that's why I'm saying that my first experience kind of influenced me even getting the follow-up the first time I noticed any slight change.

Person-related issues. Some women talked about a general discomfort or being uncomfortable or squeamish about their breasts, thereby forgoing Breast Self Exams (BSE) and being reluctant to touch and feel their own breasts. One woman noted:

110: I'm so squeamish, I don't like examining, doing breast examination. It's just, it's a really gross feeling to touch your own breasts.

Other personal delay factors included trying to get pregnant or breast-feeding and, therefore, minimizing the symptom because it was attributed to either IVF treatment, or to changes in the breast as a result of breast-feeding. These physiological changes and significant shifts to the reproductive system, which included changes in breast size and density, led some women to delay seeking care for their breast symptoms. One participant explained:

109: Yeah, well in that period, in the period from 28-36, there were lots of [reproductive related] things medically going on with me, primarily in trying to get pregnant.... I was using fertility drugs... I got pregnant, I had an ectopic pregnancy, and I had chicken pox while I was pregnant. You know there were just so many other things going on that, you know, I had a miscarriage.

Another young woman dismissed her breast symptom because she thought she was too young to have breast cancer. She said:

113: I really didn't believe that at 32, I could possibly have breast cancer.

Finally, some women avoided seeking care because of the cultural stigma around breast cancer. One participant thought that having a diagnosis of cancer meant that people would take advantage of you, feel sorry for you, or think you are contagious, dangerous, or unlucky. One participant explained:

114: I did not want to ask him [the doctor about symptom]. For many reasons from old country... [if sick] they will not help me with my property... and other people... if they know you because they "ah she got cancer now, forget it now, don't help her"... Maybe people they stay away from you ... they don't want to be close to you, they don't want to come to your house.

\section{Family physician explanations for delay}

The physician informants named a number of different reasons for why they assumed women delayed seeking care for their breast cancer symptoms. These explanations mostly fell into the personal attribution category in reference to the patient's lives or personalities (See Table 3 for an outline of these themes). These included: addiction history, fatalistic thinking in the patient, patient seeing a holistic doctor, mental health issues or personality traits, patient having a history of sexual abuse or feelings of guilt. These findings are further distilled below.
An addiction history, or shame around substance abuse as contributing to delay in seeking medical care was noted by one physician:

202: She had a long history of depression and alcoholism... it might have had to do with the shame of the alcoholism or the shame of having waited... she waited months.

Fatalistic thinking, or the patient thinking they are going to die from cancer no matter what one does and, therefore, avoiding medical intervention all together was another theme reported by physicians.

205: She was so convinced she had cancer, but hadn't come because she wasn't convinced the treatment would help her and so she thought she was dying.

Some patients opted to see a holistic doctor for care instead of coming to family physician first.

202: She went to a holistic doctor. And apparently he told her that this was, that it has something to do with having been hit in the breast at some point by an ex-husband who was abusive and that it was just stuff coming out, so by the time she presented to me, she was very advanced, in fact, died of it quite quickly.

Certain mental health issues and personality traits suggesting a high need for control were identified as contributing to delay.

208: There was a lot of other stuff going on in her life, psychological things... she was highly anxious... there were some mood-related issues... anxiety, mood, family stresses, those kinds of things combined you know she wouldn't always follow up on the things that I would place priority on.

Having a history of sexual abuse as contributing to delay was also identified.

202: I had a woman who had been abused by her father. And the form of abuse had been fondling her breasts. So when her breasts started to kind of shrivel, she was really pleased. And she did nothing about it until it ulcerated.

Some physicians described patients feeling guilty about not acting right away on an ambiguous symptom and, thus, delaying treatment further.

208: It was clear to the nurse that she felt guilty about having delayed... she had felt changes in her breast in an ongoing basis.... she realized there was something there, she realized that she should have come in and hadn't gotten around to it.

\section{Discussion}

Examining the narratives of patients and physicians revealed that women and doctors sometimes hold different explanations as to why women delay seeking care for their breast symptoms. While the women in this study focused on both doctor and personal aspects (i.e., feeling dismissed in the past by a physician and having competing, hard-to-diagnose health demands) and personal

\section{Table 3. Doctor narratives around explanations for delay}

- Addiction history and shame around substance abuse

- Fatalistic thinking

- Preferentially attending holistic doctor

- Mental health issues/personality traits

- History of sexual abuse

- Guilt around health care behaviour 
situational explanations (i.e., being uncomfortable with breasts, pregnancy and breastfeeding issues, and having a cultural stigma around a cancer diagnosis), family physicians tended to focus on different things. Interestingly, physicians were more likely to name personal aspects in relation to the women's lives or personality traits in their explanations (i.e., the patient having addiction history, having a family history with breast cancer, employing fatalistic thinking, patient seeing a holistic doctor and the patient feeling guilty) than health care factors.

Taking a narrative approach means listening to people's stories as they arise from their own perspective and not as a history of facts (Mishler, 1986a, 1986b). The Travelling on Parallel Tracks analogy captures and highlights the instances where the physicians and the patients had different explanatory paradigms and this has implications for the understanding and prevention of delay. (See Heisey et al., 2011, for a report on the findings of instances where the physicians and the patients agreed on the reasons for delay.)

Physician-patient communication. While there are many possible interventions to improve help-seeking behaviour, one particularly pertinent goal would be to improve communication between family physicians and patients. Ellingson and Buzzanell (1999) suggest that improving such communication can be used to "a) elicit patient concerns, particularly on sensitive issues that patients find uncomfortable or difficult to discuss; (b) maximize patient satisfaction; (c) improve preventative medicine; (d) produce more accurate diagnosis; (e) increase patient compliance" (p. 154). In the context of delay where the goal is to encourage women to seek consultation with their family physician as quickly as possible, improving communication, as illustrated in the above five goals, can potentially be very effective in meeting the aims of encouraging early help-seeking behaviour.

One way in which to apply these findings is to encourage FPs and other health care professionals such as family practice nurses involved in front-line work to inquire about the historical and contextual aspects of women's lives (Andersen, Vedsted, Olesen, Bro, \& Sondergaard, 2009). By being sensitized to the issues that women care about, physicians and nurses might be able to engage conversation around delay related factors before a breast symptom appears and, therefore, pre-empt delay in seeking care. For example, an FP or a family practice nurse might conduct an intake history of women's previous experiences within the health care system to assess whether she is skeptical about the level of care she expects to receive because of previous negative experiences. The FP or nurse can also inquire about a woman's comfort level with her own breasts and the degree to which she participates in breast awareness.

As with our own findings, other research has explicitly made the link between physician-patient communication and health care avoidance. Cobb and colleagues (1954) found that cancer patients who delayed seeking treatment had reported negative experiences with physicians in the past. Moore and his colleagues (2004) similarly found that the way people had been treated by health care providers in the past determined whether they sought treatment in the future for a range of health care issues, even among those who were young, insured, and familiar with health care settings. By focusing on improving patient-physician communication overall in the broader context of breast symptoms and delay, physicians and nurses in all primary care settings might increase the likelihood that women will seek help sooner in their practice than they would otherwise.

Health care professional neutrality. While the women's narratives point to the need for physicians and other health care professionals including primary and oncology nurses to take a more thorough, historical, and contextual approach in communication long before a breast symptom appears, the physician's narratives point to the need for less value-laden attributions around delay. We stress again that we, ourselves, are not making judgments about the "right" explanations; it may be that the physician's attributions are factually correct about reasons women delay seeking their care. The goal, however, is not to uncover the "truth" about what happened, but to improve help-seeking behaviour among patients by listening to patient and physician narratives. In this context, it is noteworthy that no patients talked about lifestyle or personality factors as having caused their delay such as mental health issues, feelings of guilt, or employing fatalistic thinking. This may be because the women were less likely to self-report these conditions due to associated stigma and shame. Indeed, it is that same stigma that may stop a patient from talking to their physician or nurse about breast cancer symptoms, pointing to the need for a neutral, non-judgmental environment to encourage disclosure of such sensitive information.

Our findings suggest that patients and family physicians can be on parallel tracks when it comes to understanding why some women delay seeking care for their breast cancer symptoms. These findings are relevant to all health care professional settings. When caring for this subpopulation of breast cancer patients, it is sometimes difficult for members of the oncology health care team to understand what triggers a woman to seek care when they do. It is the oncology nurse who tends to establish a primary relationship with the patient when they enter the cancer system. Understanding the importance of exploring women's histories using a supportive and nonjudgmental approach is crucial to establishing a therapeutic relationship at a time when they may be feeling ashamed, disappointed, or angry for not presenting earlier. This might also facilitate earlier help-seeking behaviour with future health care issues, as they undergo primary treatment for their newly diagnosed breast cancer. Improving patient and physician communication and encouraging family physicians and all health care professionals to adopt a more reflexive and broad perspective when attempting to understand delay behaviour, may help lead patients and doctors to a shared or convergent track.

\section{REFERENCES}

Andersen, B.L., Cacioppo, J.T., \& Roberts, D.C. (1995). Delay in seeking a cancer diagnosis: Delay stages and psychophysiological comparison processes. British Journal of Social Psychology, 34, 33-52.

Andersen, R.S., Vedsted, P., Olesen, F., Bro, F., \& Sondergaard, J. (2009). Patient delay in cancer studies: A discussion of methods and measures. BMC Health Services Research, 9, 189.
Arndt, V., Sturmer, T., Stegmaier, C., Ziegler, H., Becker, A., \& Brenner, H. (2003). Provider delay among patients with breast cancer in Germany: A population-based study. Journal of Clinical Oncology, 21(8), 1440-1446.

Beattie, A. (2009). Detecting breast cancer in a general practice: Like findings needles in the haystack? Australian Family Physician, 38(12), 1003-1006. 
Bish, A., Ramirez, A., Burgess, C., \& Hunter, M. (2005). Understanding why women delay in seeking help for breast cancer symptoms. Journal of Psychosomatic Research, 58, 321-326.

Bruner, J. (1987). Life as narrative. Social Research, 54, 11-32.

Bruner, J. (1990). Acts of meaning. London: Harvard University Press.

Caplan, L., \& Helzlsouer, K. (1992-1993). Delay in breast cancer: A review of the literature. Public Health Review, 20, 187-214.

Cobb, B., Clark, R.L., McGuire, C., \& Howe, C.D. (1954). Patientresponsible delay of treatment in cancer, a social psychological study. Cancer, 7, 920-926.

De Nooijer J., Lechner L., \& De Vries, H. (2001). Help-seeking behaviour for cancer symptoms: Perceptions of patients and general practitioner. Psych-Oncology, 10, 469-478.

Davis, E. (2008). Risky business: Medical discourse, breast cancer, and narrative. Qualitative Health Research, 18, 65-76.

Ellingson, L.L., \& Buzzanell, P.M. (1999). Listening to women's narratives of breast cancer treatment: A feminist approach to patient satisfaction with physician-patient communication. Health Communication, 11, 153-183.

Facione, N.C., Miaskowski, C., Dodd, M.J., \& Paul, S.M. (2002). The self-reported likelihood of patient delay in breast cancer: New thoughts for early detection. Preventive Medicine, 34, 397407.

Fergus, K.D., Fitzgerald, B., Granek, L., Clemons, M., Zalany, L., \& Eisen, A. (2011). Knowing and disclosure processes in the symptom appraisal of breast cancer: A qualitative analysis of relationship factors influencing presentation for medical evaluation. Journal of Health Psychology, 16(4), 653-666.

Granek, L., \& Fergus, K.D. (2011). Resistance, agency, and liminality in women's accounts of help-seeking upon discovery of an abnormal breast symptom. Manuscript in preparation.
Heisey, R., Clemons, M., Granek, L., Fergus, K., Hum, S., Lord, B., McCready, D., \& Fitzgerald, B. (2011). Health care strategies to promote earlier presentation of symptomatic breast cancer: Perspectives of women and family physicians. In Press in Current Oncology.

Meechan, G., Collins. J., \& Petrie, K.J. (2003). The relationship of symptoms and psychological factors to delay in seeking medical care for breast symptoms. Preventative Medicine, 3, 374-378.

Miles, M., \& Huberman, A. (1994). Qualitative data analysis, 2nd ed. Thousand Oakes, CA: Sage Publications.

Mishler, E.G. (1986a). The analysis of interview narratives. In T. Sarbin (Ed.), Narrative psychology: The storied nature of human conduct (pp. 233-255). New York: Praeger.

Mishler, E.G. (1986b). Research interviewing: Context and narrative. Cambridge, MA: Harvard University Press.

Moore, P., Sickel, A., Malat, J., Williams, D., Jackson, J., \& Adler, N. (2004). Psychosocial factors in the medical and psychological treatment of avoidance: The role of the doctor-patient relationship. Journal of Health Psychology, 9, 421-433.

Nosarti, C., Crayford, T., Roberts, J.V., Elias, E., McKenzie, K., \& David, A.S. (2000). Delay in presentation of symptomatic referrals to breast clinic: Patient and system factors. British Journal of Cancer, 82(3), 742-748.

O’Mahoney, M., \& Hegarty, J. (2009). Help seeking for cancer symptoms: A review of the literature. Oncology Nursing Forum, 36(4), E178-84.

Richards, M., Smith, P., Ramirez, A., Fentiman, I., \& Rubens R. (1999). The influence on survival of delay in the presentation and treatment of symptomatic breast cancer. British Journal of Cancer, 79(5-6), 858-64.

White, H. (1981). The value of narrativity in the representation of reality. In W.J.T. Mitchell (Ed.), On narrative (pp. 1-23). Chicago, IL: University of Chicago Press.

\section{Appendix A: Interview guidelines}

Probing interviews with women:

1. Tell us your story of how you came to be seen here.

2. Do you recall when you first noticed a change in your breast?

3. Do you recall when you first thought it might be cancer? Why?

4. Do you recall when you first confided in a friend or relative?

5. Do you recall your emotions around this experience?

6. Do you recall your first visit to your family physician? Your feelings at that time?

7. Is there anything we could have done differently that would have made it easier to seek care?

8. What changes would you like to see in our health care system that would help women with similar symptoms to seek care?

- Information-what would help (from whom and how)?

- Support

- Empowerment

- Accessibility

- Stigma

9. What do you think your family physician might do to encourage other women with similar symptoms to seek help? 10.Anything he or she could say?

\section{Probing interviews with physicians:}

1. Can you recall a patient who presented three months or more after detection of her breast cancer symptoms?

2. Tell us her story.

3. What were your feelings at that time?

4. Why do you think it took her so long to present?

5. Can you think of anything that might have helped her seek help sooner?

6. In hindsight, did you have any idea that she had a breast problem earlier?

7. Are there any characteristics of this patient that you feel enhanced the delay?

- Personality traits

- Living arrangements

- Life demands

- "Not wanting to bother the doctor"

- History of abuse

8. What changes would you like to see in our health care system to help women with similar symptoms seek care?

- Information

- Support

- Empowerment

- Accessibility

- Stigma

9. Is there anything you would have done differently if you could go back in time? 